\title{
Improving Vertical Handoffs Using Mobility Prediction
}

\author{
Mahmoud Al-Ayyoub, Ghaith Husari and Wail Mardini \\ Jordan University of Science and Technology \\ Irbid, Jordan
}

\begin{abstract}
The recent advances in wireless communications require integration of multiple network technologies in order to satisfy the increasing demand of mobile users. Mobility in such a heterogeneous environment entails that users keep moving between the coverage regions of different networks, which means that a non-trivial vertical handoff scheme is required in order to maintain a seamless transition from one network technology to another. A good vertical handoff scheme must provide the users with the best possible connection while keeping connection dropping probability to the minimum. In this paper, we propose a handoff scheme which employs the Markov model to predict the users' future locations in order to make better handoff decisions with reduced connection dropping probability and number of unnecessary handoffs. Through simulation, the proposed scheme is compared with the SINR-based scheme, which was shown to outperform other vertical handoff schemes. The experiments show that the proposed scheme achieves significant improvements over the SINR-based scheme that can reach $51 \%$ in terms of the number of failed handoffs and $44 \%$ in terms of the number of handoffs.
\end{abstract}

Keywords-Heterogeneous wireless networks, Vertical handoff, Markov model, Artificial intelligence, Mobility management.

\section{INTRODUCTION}

Even before the first commercial adoption of cellular network in North America and Western Europe in the late 70s and early 80s, such networks received a great deal of attention from researchers in both academia and industry [14]. The recent wide spread of smart phones and tablets allowed people to connect to the Internet easily. Coupled with the increase in the demand for online multimedia material and Voice over IP (VoIP) applications, the need to maintain a "high quality" connection to the Internet anywhere and anytime has become a very important issue. This is achieved through the use of multiple wireless access technologies. One example are the Wireless Local Area Networks (WLANs), which are widely deployed because they are cheap to deploy and provide high bandwidth. However, contrary to Wide Code Division Multiple Access (WCDMA) networks, WLANs have very low service mobility due to its shorter transmission range with limited area of coverage. This fosters the necessity for collaboration between heterogeneous wireless networks such as WLANs, Wide Code Division Multiple Access (WCDMA), and other network variations. Users, or Mobile Terminals (MTs), have different service needs regarding the connection bandwidth, monetary cost, security, etc. Also, MTs move from one location to another that might be outside the area of coverage of the preferred wireless network currently serving the MTs. So, a MT should get the best possible connection that suits its needs

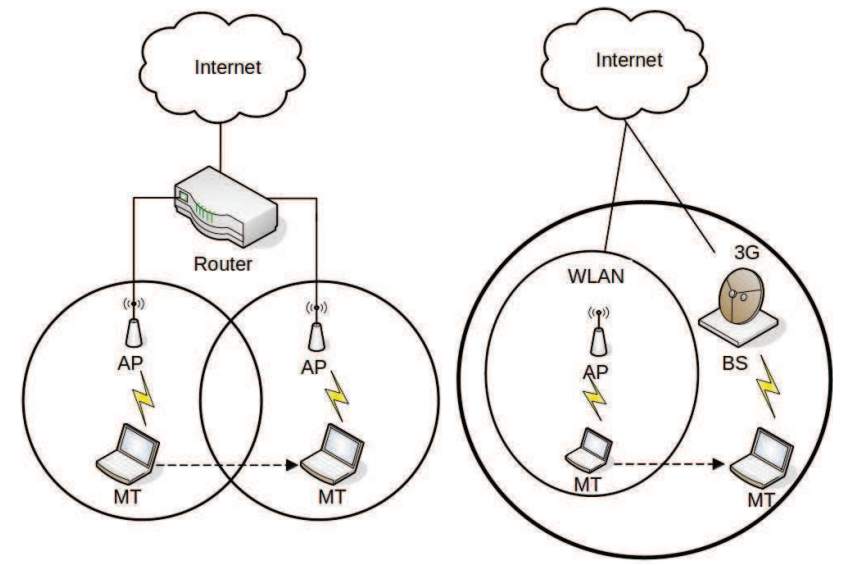

Fig. 1: The two types of handoff: horizontal (left side) and vertical (right side).

and maintains the Always Best connected (ABC) concept as it affects the Quality of Service (QoS) of the connection [8].

Different access technology devices have different transmission power levels and thus different areas of coverage. When a MT starts moving away from its connection-providing device, another device that covers the new location of the MT is needed. The process of moving a MT's communication session from an access technology device to another in order to ensure the continuity of the connection without affecting the ongoing session is called handoff [18], [21]. Two types of handoff are commonly discussed in the literature: (i) horizontal handoff, which occurs when the MT moves from one access point to another within the same wireless technology, and (ii) vertical handoff, which occurs when the MT moves from one access point to another access point that belongs to different wireless technology. Figure 1 shows an example for each of these two types. It is noteworthy to mention that the focus of this work is on the more challenging case where the MT has a list of handoff candidates and it must choose the best one wisely in order to maintain its communication session while trying to achieve higher QoS.

As a MT starts moving away from the service providing device, the Received Signal Strength (RSS) gets weaker and the service quality starts to degrade. So, at a certain point, the MT must change its association and connect to a new access point that can provide better service (e.g., more bandwidth, stronger signal strength, etc.) without the loss of the ongoing call or connection. In the literature [1], [4], there have discussions of other factors governing the handoff decision including the 
Signal to Interference plus Noise Ratio (SINR), power, delay, available bandwidth and QoS guarantees, security and financial issues. The focus of this work is on improving the reliability of the connection by reducing the probability of disconnections, which occur when MTs try to perform a handoff to an access technology device with no available channels. Disruptions of this type are generally viewed as annoying and unacceptable so much that the users are willing to accept lowered bandwidth if it means improved reliability [23]. Another objective of the proposed scheme is to minimize the number of unnecessary handoff leading to better QoS guarantees [23].

Despite the strong intuition that the geographical nature of the handoff problem plays a significant role in the success of any handoff scheme, most of the current works simply ignore it. This work is based on the idea that the knowledge of the future positions of the MT can aid in making better handoff decisions. One of the benefits gained from this is choosing a handoff destination that is usable for a longer period of time, and thus, reducing the number of unnecessary handoffs. Another benefit is realized when considering the fact that the intersections of movement trajectories of the many MTs are bound to create heavily loaded regions. Taking the future locations of the MT can allow the handoff scheme to avoid choosing the handoff candidates with such "central" locations, which helps in balancing the load in the network as well as decreasing the disconnection probability. The experiments discussed in Section IV support these intuitive arguments as to why the proposed scheme outperforms other schemes such as the SINR-based scheme.

The rest of the paper is organized as follows. The following section surveys the recent works in the literature on vertical handoff schemes while Section III describes the system model used in this work, the assumptions made and the proposed scheme. The performance of the proposed scheme is evaluated in Section IV and the paper is concluded in Section V.

\section{RELATED WORKS}

Due to its importance to the increasingly spreading heterogenous wireless networks, the vertical handoff problem has been studies extensively. Below, we review some of the most important schemes proposed in the literature. We categorize the covered schemes based on their general approach.

We start our coverage with one of the basic techniques which focusing on Received Signal Strength (RSS) as the main factor in the handoff decision. An example of such techniques is the adaptive lifetime-based vertical handoff (ALIVE-HO) scheme proposed by Zahran et al. [25], [24]. While considering delay, authentication, and service initiation, the ALIVE-HO scheme used the RSS to estimate how long a MT's needs can be served by a WLAN which helped in the handoff decision. The objectives were to reduce the number of dropped connections and increase the connection duration by delaying the handoff. According to simulation results shown by the authors, this lead to reducing unnecessary handoffs while increasing the average throughput. Moreover, the proposed scheme is able to adapt to the application requirements and user mobility by using the lifetime metric. In a similar work, Yan et al. [19], [20] used RSS measurements to estimate how long a MT will stay in a WLAN cell (i.e., the traveling distance within the cell). These estimations were exploited in a vertical handoff scheme with reduced number of unnecessary handoffs and improved overall network utilization. The experiments conducted showed that their algorithm reduced the probability of handoff failures as well as unnecessary handoffs provided that the estimated traveling distance is smaller than a certain threshold. Finally, Mohanty et al. [12] proposed a vertical handoff scheme that uses MTs speed and handoff signaling delay to improve the handoff process WLANs and $3 \mathrm{G}$ cellular networks.

A closely related criterion to RSS is the signal to interference plus noise ratio (SINR). The vertical handoff scheme of Ayyappan et al. [3] used SINR as the main criterion in the handoff decision. Using Shannon's capacity theorem, the SINR values of each network were used to compute the throughput of each network. The network with the best QoS was selected. The experiments conducted by the authors showed that the SINR-based scheme outperforms other the RSS-based schemes in terms of the throughput and the number of dropped connections. Yang et al. [22] proposed a multi-dimensional adaptive SINR based vertical handoff scheme (MASVH) scheme, which incorporated many aspects into the handoff decision including the SINR, the required user bandwidth, the traffic cost and the network utilization. The conducted experiments showed that MASVH improved the network throughput and decreased both the probability of failed handoffs and the cost of traffic.

In the last scheme of the previous paragraphs, the authors employed (among many factors) the available bandwidth as a factor in the handoff decision. This is not the only work with this basic idea. A QoS-based vertical handoff scheme between WLANs and Wireless Wide Area Networks (WWANs) was proposed by Ayyappan and Kumar [2] which takes into account the available bandwidth as well as the QoS requirements of the user. Another similar scheme for vertical handoff between WLANs and WCDMA networks was proposed by Yang et al. [23]. They key idea in this scheme was to use the SINR values to estimate the achievable bandwidths at the candidate handoff networks in order to make QoS-aware handoff decisions.

As mentioned in Section I, despite the strong intuition that the geographical nature of the handoff problem plays a significant role in the success of any handoff scheme, most of the current works simply ignore it. However, there are a few works (such as [10], [26], [4]) that took geographical information into account while performing the vertical handoff decision. In [10], the locations of the handoff candidates were used to repeatedly compute the distances between them and the MT's position. The handoff decision is triggered when the MT determines that it is moving away from its current access technology device (which can be easily determined if the MT maintains the history of its recent few positions) and the chosen candidate is the closest one to the MT's current position provided that it is closer than current access technology device. Another location-based scheme was proposed by Zhang et al. [26], where dynamic programming is used to utilize the MT's current location and mobility to improve the handoff decision.

Since the proposed handoff scheme utilizes prediction methods to improve the handoff decision, it is important to cover works following a similar approach. Becvar [5] proposed 
a new scheme to perform handover based on prediction that uses the history of handover between every two adjacent base stations. With repetitive handovers from a certain base station to an adjacent one, future handovers of mobile terminals that arrive to the base station could be predicted based on the frequency of handovers history between pairs of base stations. The proposed method needs time to adapt and calculate handover probability from a base station to another based on handover history. The paper states that the model needs 2000 to 3000 seconds, which corresponds to 4000 to 6000 handovers [5]. The model must know its environment which consists of neighboring base stations. So, whenever a handover occurs from a base station to another, it affects the probability of handover between that pair of base stations in a positive manner and other base stations handoff probability in a negative manner (if one option probability is increased, other available options probability is decreased). The simulation results show that the prediction hit rate is high when very repetitive handover between a pair of base stations. However, when there are many neighbors and the handover history is distributed on all neighboring base stations, the prediction of handover drops. The prediction hit rate in all scenarios varies from $47 \%$ - $20 \%$ since it decreases with the increase of neighboring base stations. Finally, Chi et al. [7] suggested two criteria, the wrong decision probability (WDP) and the handover probability (HP), for evaluating handoff schemes and provided mathematical modeling for both measures.

A completely different fuzzy logic approach for vertical handoff between WLANs and Universal Mobile Telecommunications Systems (UMTS) was followed by Xia et al. [17]. The authors suggested using RSS and available bandwidth in addition to predicting the future RSS using differential prediction algorithms to trigger the handoff process. The handoff candidate is selected by the performance evaluation results of a Fuzzy logic based Normalized Quantitive Decision (FNQD).

In a more recent work exploiting fuzzy logic, Boussen et al. [6] proposed a new context aware vertical handover decision algorithm to select the best network in terms of QoS and energy efficiency. They use a fuzzy logic system to initiate handoffs based on context. However, the fuzzy logic system they use is not as accurate as artificial intelligence prediction algorithms (e.g., Markov Model).

Omheni et al. [13] proposed a new approach for handover decision making in heterogeneous wireless network to select the best network based on the application. Simulation showed that the proposed algorithm guarantees QoS requirements and reduce the blocking probability of handoff requests, which also maximizes bandwidth offered from the selected network. However, the approach required assistance from the network to provide context information in case of mobility.

Finally, Wang [15] provided performance evaluation framework for network selection strategies based on models constructed by using stochastic process algebra. The proposed framework captures the throughput rate and mobility features of the nodes in 3G-WLAN interworking networks. Evaluation is performed in terms of throughput, number of handovers, and network blocking rate. They showed that performance is very sensitive to the traffic pattern of the mobile node which affects the number of handoffs that in turn affects the throughput rate and number of blocked handoff requests (i.e., QoS). In our work, we use the same evaluation parameters to show the quality of our proposed algorithm.

\section{System Model And Proposed Solution}

Before going into the details of the proposed vertical handoff scheme, a quick coverage of the system model and assumptions made is necessary. The following subsection discusses the signal propagation model whereas Section III-B discusses the considered mobility models.

\section{A. Signal Propagation Model}

This work considers heterogenous networks consisting of two different types of access technology: WLANs and WCDMA cellular networks. As with other wireless communication technology, the main point is the Received Signal Strength (RSS). In the following paragraphs, we discuss the equations used to compute SINR and RSS for both network types under consideration [23], [24], [3], [4].

We start with WCDMA cellular networks. For a WCDMA Base Station (BS) $j$ and MT $i$, the RSS (in $\mathrm{dBm}$ ) is computed using the following equation.

$$
R S S_{C}=P_{j}+G_{j}-P L_{i j}-A_{j},
$$

where $P_{j}$ is the transmission power of $j$ (in $\mathrm{dBm}$ ), $G_{j}$ is the transmitted antenna gain (in $\mathrm{dB}$ ), $P L_{i j}$ is the total path loss (in $\mathrm{dB}$ ) as defined in Equation 2 and $A_{j}$ is the connector and cable loss (in $\mathrm{dB}$ ). To compute the total path loss for MT $i$ and $\mathrm{BS} j$, we use the following equation.

$$
\begin{aligned}
P L_{i j} & =135.41+12.49 \log \left(f_{j}\right)-4.99 \log \left(h_{j}\right) \\
& +\left(46.84-2.34 \log \left(h_{j}\right)\right) \log \left(d_{i j}\right),
\end{aligned}
$$

where $f_{j}$ is the frequency (in $\mathrm{MHz}$ ), $d_{i j}$ is the distance (in kilometers) between $i$ and $j$ and $h_{j}$ is the effective antenna height (in meters).

As for WLANs, the RSS computation is as follows. For a WLAN Access Point (AP) $j$ and MT $i$, the RSS (in $\mathrm{dBm}$ ) is computed using the following equation.

$$
R S S_{W}=P_{j}-P L_{i j}
$$

where the total path loss is computed using the following equation.

$$
P L_{i j}=L+10 n \log \left(d_{i j}\right)+S
$$

where $L$ is a constant power loss, $n$ is the path loss exponent (assumed to be between 2 and 4), and $S$ is a zero-mean normal random variable representing the effect of fading.

\section{B. Mobility Model}

In this work we use two mobility models. The pathway mobility model and a proposed variation of that model which aims to help us better understand the scheme.

Mobile nodes do not move in a completely random way, nor in a complete deterministic way as well. Instead, their movements include both random and regular components [16]. So a memoryless mobility model, that is completely random such as the random waypoint, and a deterministic mobility model (with no randomness as it completely defines the velocity and movement direction for each node in advance and 


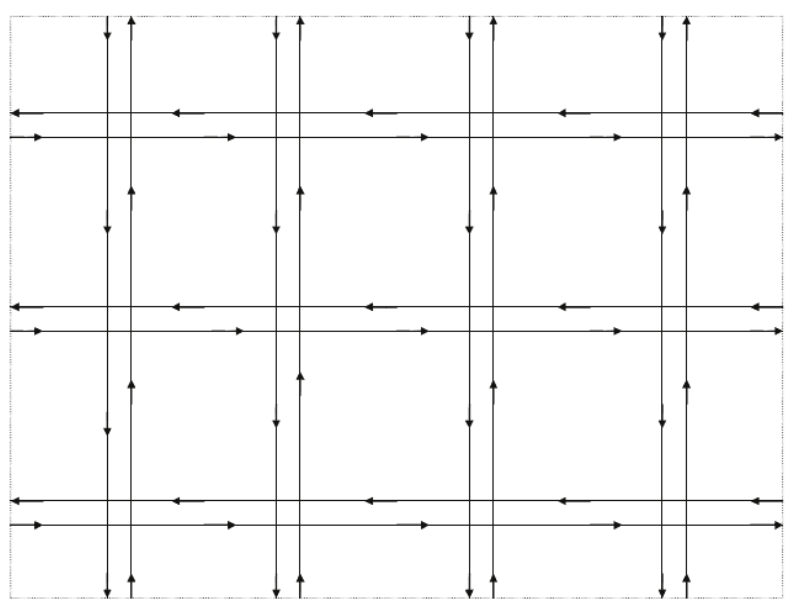

Fig. 2: The Pathway mobility model.

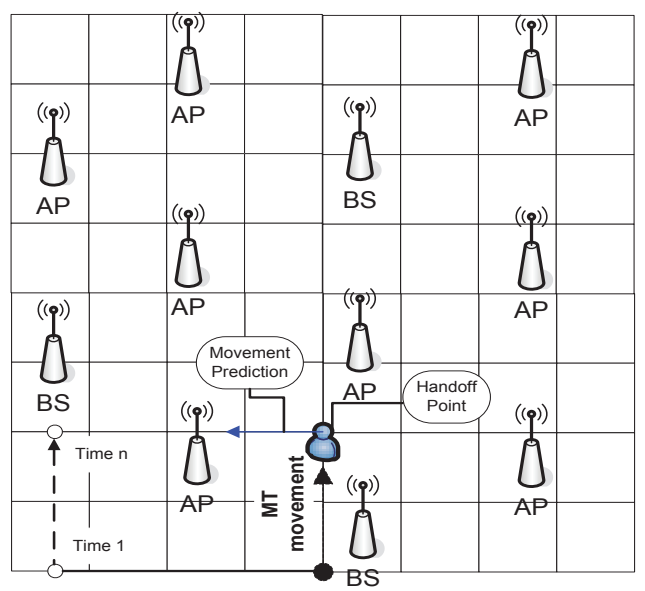

Fig. 3: The handoff process in MPVH. thus every node's movement is certain and known in advance) both are not suitable to describe users' movements inside a city. Accordingly, we describe the movements of MTs using pathway mobility model.

The pathway mobility (PW) model [11] is simple and contains both regular and random components. In this model, the MTs are randomly placed on the edges of the terrain's grid (see Figure 2). Each node randomly chooses a location as a destination point and starts moving to it through the shortest path along the edges of the grid with constant speed chosen uniformly and randomly from a velocity range $\left[v_{\min }, \ldots, v_{\max }\right]$. When the destination point is reached, the node stops for a randomly selected pause time. After the pause time is over, the node chooses another destination point and repeats the whole process until the simulation time is over. In case there are more than one shortest path, one of them is randomly selected and the mobile node will go to destination through this path.

The pathway model with straight route preference (PWSRP) is similar to the pathway way model. However, the first one picks the shortest route/way to the destination that has the least direction changes (left or right turns). In other words, this model tries to keep going straight as long as possible and avoids making left or right turns unless necessary.

\section{Mobility Prediction-Based Scheme for Vertical Handoff $(\mathrm{MPVH})$}

In this section, the Mobility Prediction-Based Scheme for Vertical Handoff (MPVH) is discussed. MPVH involves two decisions: when should a MT perform a handoff (handoff trigger) and how to do it (i.e., to which BS/AP the MT should connect). Following one of the standard techniques in the literature, a handoff is triggered solely based on the RSS. As a MT $i$ is moving away from the BS/AP $j$ to which it is connected, the RSS of $j$ at $i$ starts to decrease. At a certain point, the RSS will be too low for the communication to be successful. This will trigger the handoff. Now, the second decision related to which BS/AP the MT should handoff, is where the contribution of MPVH lies. MPVH starts by compiling a list of candidate BSs/APs with a RSS at MT $i$ above the threshold for a successful communication. MPVH then consults the prediction component (which is based on a Markov model) to compute the most probable future location of $i$ and chooses the BS/AP that is closest to this location. A similar prediction method was used in [9] to improve channel switching in cognitive radio networks. See Figure 3 for an example.

\section{Simulation Results}

In this section, simulation experiments were conducted to evaluate the performance of MPVH and compare it with one of the most widely-used vertical handoff schemes. The scheme chosen for comparison is the SINR-based scheme due to its low disconnection ratio in comparison with other schemes as mentioned in Section II.

The two performance metrics used in this comparison are as follows.

- Number of failed handoffs: when a mobile user requests to handoff to an access technology device, the request will be denied if there is no free channels at the access technology device which causes disconnection of the user. These disconnections are very annoying to mobile users. As a matter of fact, users prefer networks with higher reliability (i.e., have lower disconnection probability) to networks with higher bandwidth [23].

- Number of handoffs: Each performed handoff has an associated handoff delay (the time between the last packet received from the old access device and the first packet received from the new access device). The more handoffs performed the more handoff delay there is. This negatively affects the overall QoS [23].

The distribution of access technology devices across the network domain (terrain) directly affects the results of handoff schemes. Previous works [23], [22], [3] have distributed access technology devices across a network domain (terrain) carefully to achieve the best performance of their scheme. We use that topology to compare the MPVH scheme with 
the SINR-based scheme, in addition to another topology of uniformly distributed devices. The network consists of 7 Base Stations (BSs) and 12 Access Points (APs). The terrain size is $5000 \times 5000 \mathrm{~m}$. In our experiments, the number of MTs varies between 200 and 600. Each MT is placed on a random location and connects to the access technology device of the highest SINR value at the beginning. We use the same network configuration values shown in Table 1 of [4], which were also used in the SINR-based scheme of [23], [3].

The objective of the first experiment is to compare the performance of the two handoff schemes under consideration, MPVH and SINR-based, as the number of MTs increases. The results of this experiment (depicted in Figures 4 and 5) show a clear advantage in favor of MPVH over the SINR-based scheme in both performance metrics.

Considering the number of failed handoffs first, Figure 4(a) shows an average improvement of $35 \%$ for the pathway model (PW) and Figure 4(b) shows an average improvement of $43 \%$ for the pathway model with straight route preference (PWSRP). Such improvements can be justified as follows. The SINR-based scheme favors the access device with the best SINR value. Such devices may happen to be in "central" locations in the network that are close to the trajectories of movement for many mobile users and hence they usually have high SINR. By favoring such devices, SINR-based scheme is overloading them and possibly leading to more disconnections. On the other hand, MPVH will not favor these devices creating a more load-balanced distribution of work among access devices and reducing the disconnection probability.

As for the number of handoffs, the plots in Figure 5 show that MPVH outperforms the SINR-based scheme by an average of $33 \%$ for the PW model and $40 \%$ for the PWSRP model (as shown in Figures 5(a) and 5(b)) The interpretation of such results lies in the fact that, under the PWSRP model, MTs avoid changing their directions, which work in favor of MPVH since it uses a movement pattern capturing algorithm for the selection of the best handoff candidate.

To provide more insights into why MPVH outperforms the SINR-based scheme, consider the scenario depicted in Figure 6, where a MT is moving away from its servicing base station $\left(B S_{1}\right)$ until it reaches a handoff point (the red circle). At this point, the SINR-based scheme will handoff to the BS that has the best SINR value, which is $B S_{3}$. As the MT changes its directions and moves towards its destination, it reaches another handoff point (the green circle). Then, the SINR-based scheme will be forced to perform another handoff to the $\mathrm{BS}$ with the best SINR value at this point, which is $B S_{2}$. On the other hand, MPVH's mobility prediction component will give it a clear advantage in such scenarios. When the MT reaches the first handoff point (the red circle), MPVH will predict its future location and choose the closest base station to it as the handoff destination, which is $B S_{2}$. Now, as the MT continues its movement, the RSS value of its current servicing $\mathrm{BS}, B S_{2}$, will not drop under the threshold and a second handoff will not take place.

In the second experiment, we consider a more realistic (and more challenging) case of random network topology. In the previous experiment, the considered network topology consisted of fixed BS/AP locations as depicted in Figure 3.
Note that the BSs are placed on a triangular grid and the APs are placed in the middle of the overlap regions of the coverage areas of the BSs. Such "perfect" placement gives an advantage to the SINR-based scheme as it allows smaller overlapping regions which reduces the number of candidate handoff destinations. To study this issue, a uniform distribution of the BSs/APs is used. Figures 7(a) and 7(b) show that under such distribution, the average improvements of MPVH over the SINR-based jump to about $44 \%$ for the PW model and $51 \%$ for the PWSRP model, in terms of the number of failed handoffs. As for the number of handoffs, Figures 8(a) and 8(b) show that under such distribution, the average improvements jump to $37 \%$ for the PW model and $44 \%$ for the PWSRP model.

\section{CONCLUSion ANd Future Work}

In this work, Markov model was used to predict the future location of the user and the vertical handoffs the user will need based on that prediction. We show through simulations and experiments that predicting users' future locations provides a better tool for handoff than SINR-based scheme. This is evident by the decrease in service disconnection probability (which can reach $51 \%$ in certain cases) and the number of unnecessary handoffs (which can reach $44 \%$ in certain cases).

There are many future directions of this work. One of them is to use a higher order Markov model to improve the predictions since such model allows the exploitation of longer history of mobile nodes to predict further steps in the future. This is expected to improve the handoff decisions and therefore improve the overall system performance. In order to deal with the added complexity of such models, a compression mechanism will be necessary due the exponential nature of the Markov expansion which will burden the limited memory of a mobile node. Studying the tradeoff between the gain due to having a more accurate prediction model and the cost of maintaining such a system is an interesting future direction of this work.

\section{REFERENCES}

[1] K. Ayyappan and P. Dananjayan. RSS measurement for vertical handoff in heterogeneous network. Journal of Theoretical and Applied Information Technology, 4(10):989-994, 2008.

[2] K. Ayyappan and R. Kumar. QoS based vertical handoff scheme for heterogeneous wireless networks. Proceedings of the International Journal of Research and Reviews in Computer Science (IJRRCS'10), 1(1):1-6, 2010.

[3] K. Ayyappan, K. Narasimman, and P. Dananjayan. SINR based vertical handoff scheme for QoS in heterogeneous wireless networks. In Future Computer and Communication, 2009. ICFCC 2009. International Conference on, pages 117-121. IEEE, 2009.

[4] Abdelnasser Banihani, Mahmoud Al-Ayyoub, and Ismail Ababneh. A direction-based vertical handoff scheme. Journal of Emerging Technologies in Web Intelligence, 5(3), 2013.

[5] Z. Becvar. Efficiency of handover prediction based on handover history. Journal of Convergence Information Technology, 4(4):41-47, 2009.

[6] Sinda Boussen, Nabil Tabbane, Sami Tabbane, and Francine Krief. A context aware vertical handover decision approach based on fuzzy logic. In Communications and Networking (ComNet), 2014 International Conference on, pages 1-5. IEEE, 2014.

[7] C. Chi, X. Cai, R. Hao, and F. Liu. Modeling and analysis of handover algorithms. In Global Telecommunications Conference, 2007. GLOBECOM'07. IEEE, pages 4473-4477. IEEE, 2007. 


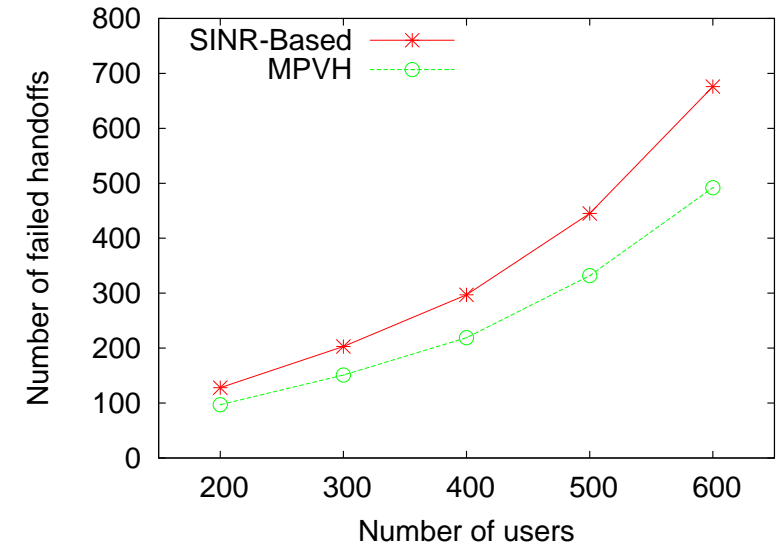

(a) PW model

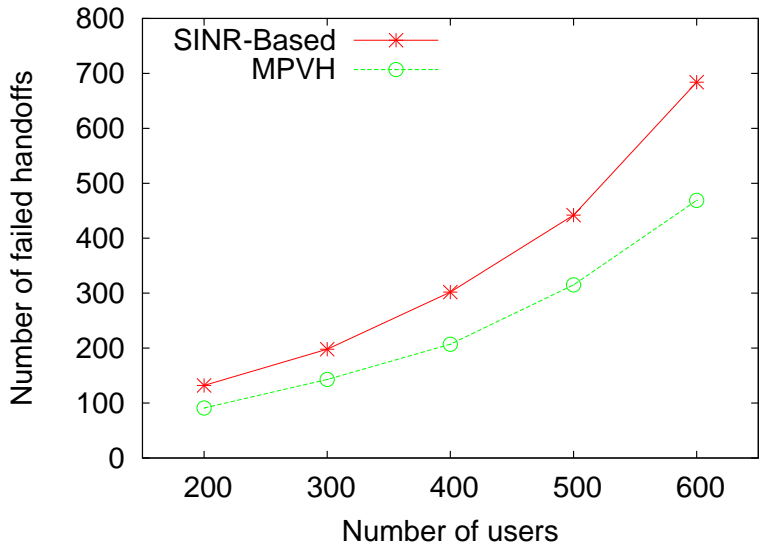

(b) PWSRP model

Fig. 4: Comparison of the number of failed handoffs by MPVH and SINR-based schemes under a fixed topology.

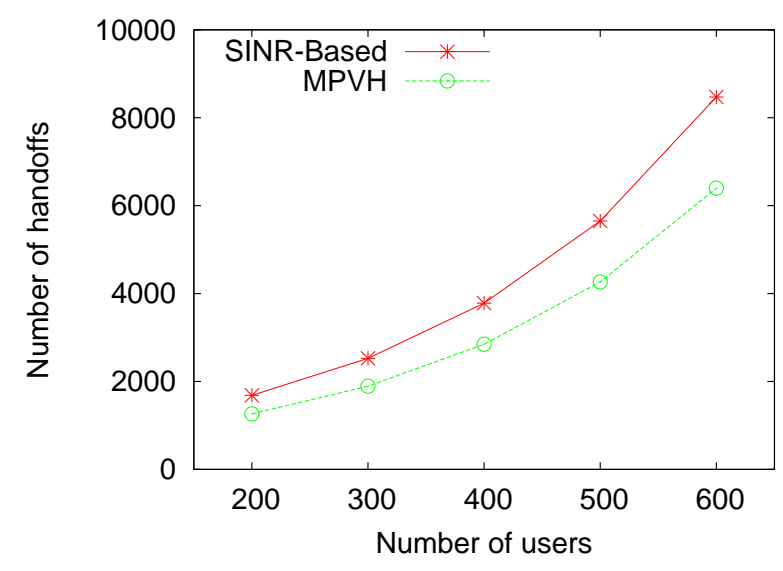

(a) PW model; Fixed topology

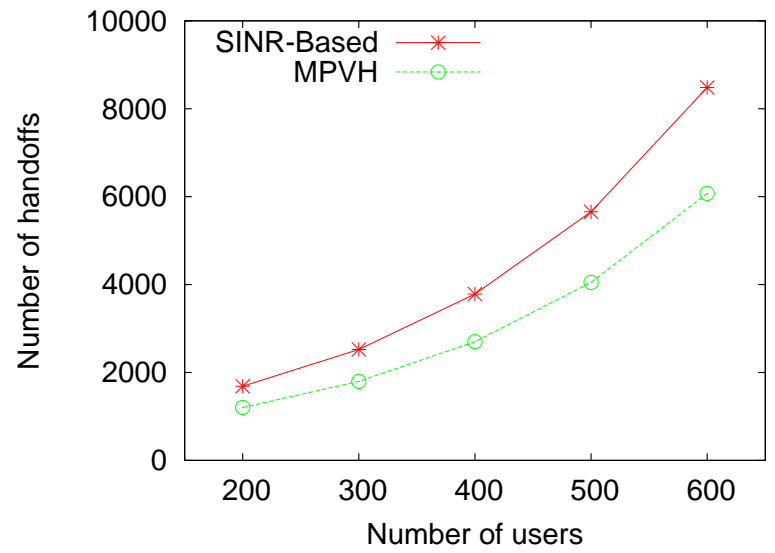

(b) PWSRP model; Fixed topology

Fig. 5: Comparison of the number of handoffs by MPVH and SINR-based schemes under a fixed topology.

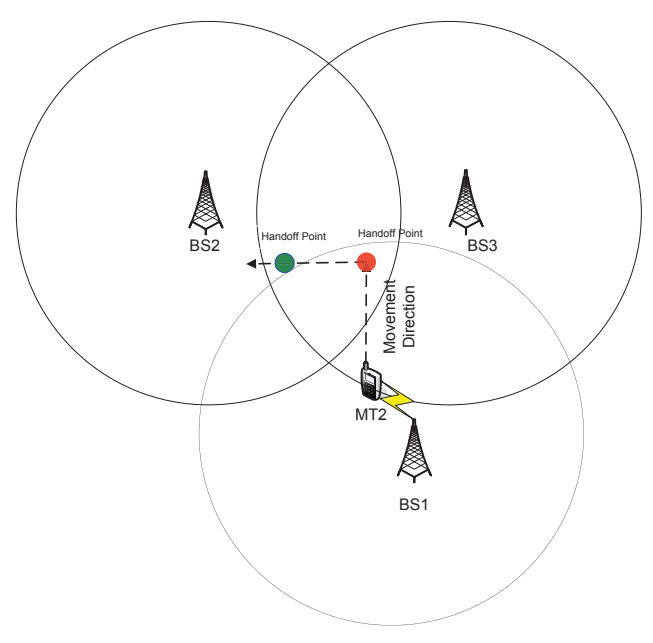

Fig. 6: A case in which MPVH outperforms the SINR-based scheme.
[8] E. Gustafsson and A. Jonsson. Always best connected. Wireless Communications, IEEE, 10(1):49-55, 2003.

[9] E. Kanan, G. Husari, M. Al-Ayyoub, and Y. Jararweh. Towards improving channel switching in cognitive radio networks. In Information and Communication Systems (ICICS), 2015 6th International Conference on, pages 280-285, April 2015.

[10] M. Lott, M. Siebert, S. Bonjour, D. von Hugo, and M. Weckerle. Interworking of WLAN and 3G systems. Communications, IEE Proceedings-, 151(5):507-513, 2004.

[11] J.G. Markoulidakis, G.L. Lyberopoulos, D.F. Tsirkas, and E.D. Sykas. Mobility modeling in third-generation mobile telecommunications systems. Personal Communications, IEEE, 4(4):41-56, 1997.

[12] S. Mohanty and I.F. Akyildiz. A cross-layer (layer 2+3) handoff management protocol for next-generation wireless systems. Mobile Computing, IEEE Transactions on, 5(10):1347-1360, 2006.

[13] Nouri Omheni, Faouzi Zarai, Mohammad S Obaidat, and Kuei-Fang Hsiao. A novel vertical handoff decision making algorithm across heterogeneous wireless networks. In Computer, Information and Telecommunication Systems (CITS), 2014 International Conference on, pages 1-6. IEEE, 2014.

[14] V Tabataba Vakili and A Aziminejad. A new dynamic channel allocation scheme based on compact pattern concept with pattern restoration. IRANIAN JOURNAL OF SCIENCE AND TECHNOLOGY, 28(B3):373382, 2004. 


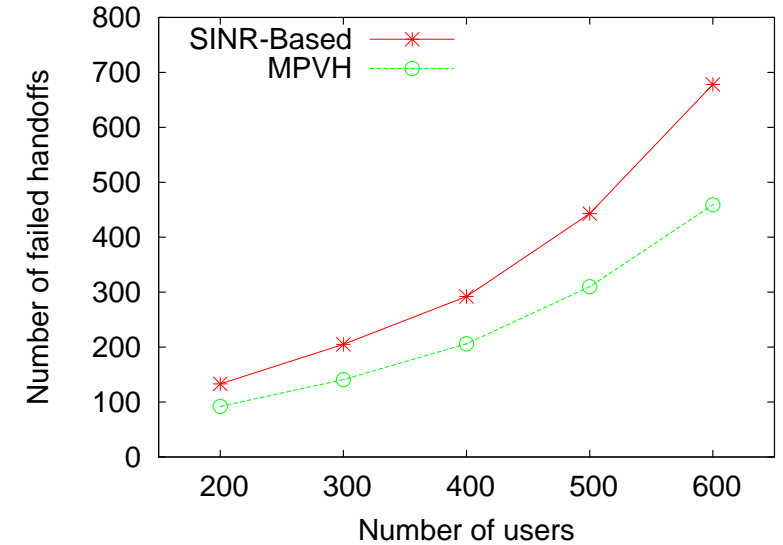

(a) PW model; Fixed topology

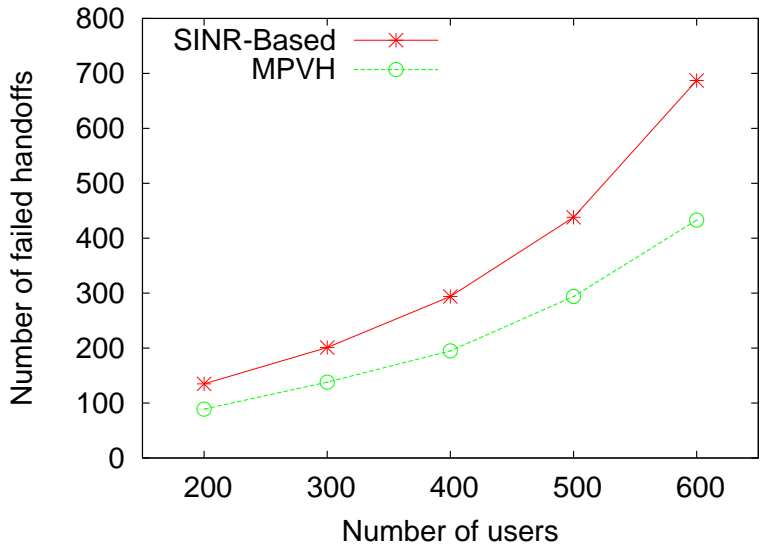

(b) PWSRP model; Fixed topology

Fig. 7: Comparison of the number of failed handoffs by MPVH and SINR-based schemes under a random topology.

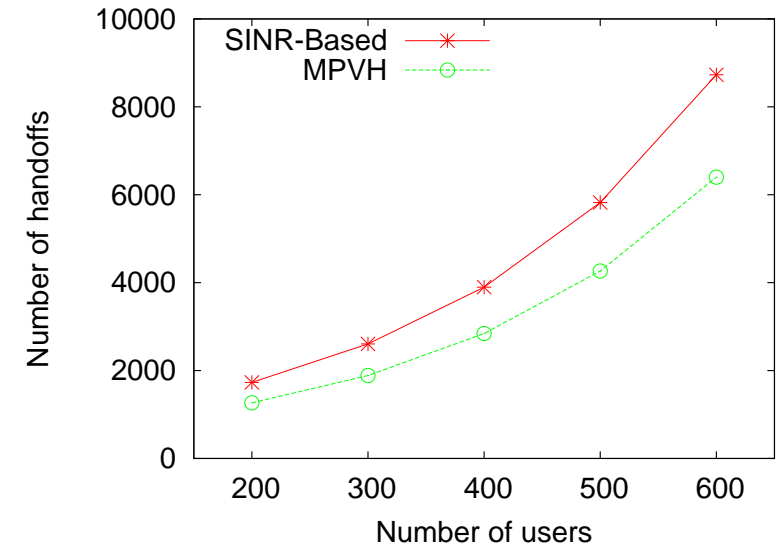

(a) PW model; Random topology

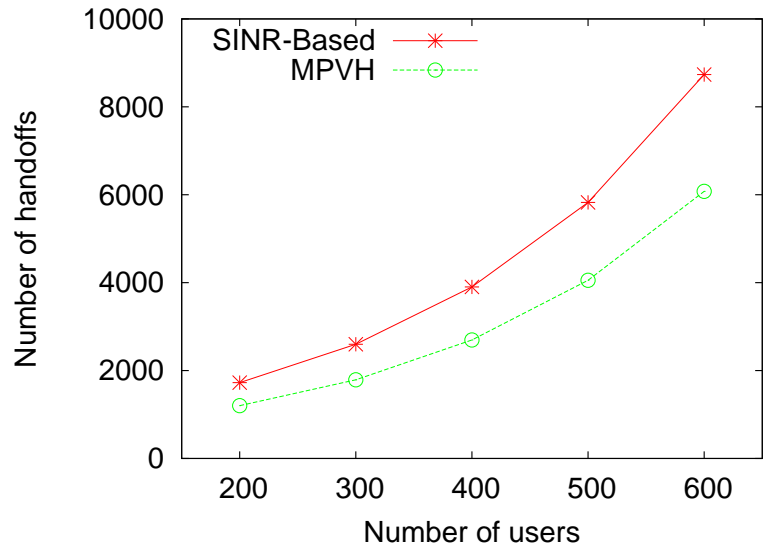

(b) PWSRP model; Random topology

Fig. 8: Comparison of the number of handoffs by MPVH and SINR-based schemes under a random topology.

[15] Hao Wang, David I Laurenson, and Jane Hillston. A general performance evaluation framework for network selection strategies in $3 \mathrm{~g}$ wlan interworking networks. Mobile Computing, IEEE Transactions on, 12(5):868-884, 2013.

[16] T.A. Wysocki, A. Dadej, and B.J. Wysocki. Advanced wired and wireless networks, volume 26. Springer, 2004.

[17] L. Xia, L. Jiang, and C. He. A novel fuzzy logic vertical handoff algorithm with aid of differential prediction and pre-decision method. In Communications, 2007. ICC'07. IEEE International Conference on, pages 5665-5670. IEEE, 2007.

[18] X. Yan, Y. Ahmet Şekercioğlu, and S. Narayanan. A survey of vertical handover decision algorithms in fourth generation heterogeneous wireless networks. Computer Networks, 54(11):1848-1863, 2010.

[19] X. Yan, N. Mani, and YA Cekercioglu. A traveling distance prediction based method to minimize unnecessary handovers from cellular networks to WLANs. Communications Letters, IEEE, 12(1):14-16, 2008.

[20] X. Yan, Y.A. Sekercioglu, and N. Mani. A method for minimizing unnecessary handovers in heterogeneous wireless networks. In World of Wireless, Mobile and Multimedia Networks, 2008. WoWMoM 2008. 2008 International Symposium on a, pages 1-5. IEEE, 2008.

[21] Xiaohuan Yan, Y Ahmet Sekercioglu, and Sathya Narayanan. Optimization of vertical handover decision processes for fourth generation heterogeneous wireless networks. In IWCMC, pages 1383-1390, 2011.
[22] K. Yang, I. Gondal, and B. Qiu. multi-dimensional adaptive SINR based vertical handoff for heterogeneous wireless networks. Communications Letters, IEEE, 12(6):438-440, 2008.

[23] K. Yang, I. Gondal, B. Qiu, and L.S. Dooley. Combined SINR based vertical handoff algorithm for next generation heterogeneous wireless networks. In Global Telecommunications Conference, 2007. GLOBECOM'07. IEEE, pages 4483-4487. IEEE, 2007.

[24] A.H. Zahran and B. Liang. Performance evaluation framework for vertical handoff algorithms in heterogeneous networks. In Communications, 2005. ICC 2005. 2005 IEEE International Conference on, volume 1, pages 173-178. IEEE, 2005.

[25] A.H. Zahran, B. Liang, and A. Saleh. Signal threshold adaptation for vertical handoff in heterogeneous wireless networks. Mobile Networks and Applications, 11(4):625-640, 2006.

[26] J. Zhang, H.C.B. Chan, and V.C.M. Leung. WLC14-6: A location-based vertical handoff decision algorithm for heterogeneous mobile networks. In Global Telecommunications Conference, 2006. GLOBECOM'06. IEEE, pages 1-5. IEEE, 2006. 